\title{
Rôle et traitement de l'obstruction nasale dans le ronflement
}

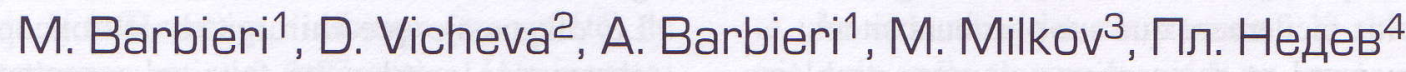 \\ ${ }^{1}$ CHU ORL Hôpital San Martino, Génes, Italie \\ 2 ORL Hôpital, Medical University, Plovdiv, Bulgarie \\ ${ }^{3}$ ORL Clinic "St. Petka" - Varna, Bulgarie \\ ${ }^{4}$ Medical University - Varna, Bulgarie
}

\section{Résumè}

L'obstruction nasale peut avoir un rôle important dans la genèse des troubles respiratoires du sommeil. En effet, en cas d'obstruction nasale une augmentation de la pression négative endoluminale s'associe à une réduction du tonus physiologique musculaire pharyngé, ce qui provoque un collapsus majeur des parois pharyngées. Etant donné le rôle joué par le nez dans la genèse et la persistance du ronflement, nous considérons fondamental d'ajouter de manière systématique à un examen fibroendoscopique avec manoeuvre de Muller l'étude de la fonction nasale au moyen de la rhinomanométrie antérieure active avec test de position et rhinométrie acoustique. Plusieurs études reportent la valeur de la chirurgie nasale pour l'amélioration de la ventilation nasale et la réduction des troubles respiratoires du sommeil même si, dans tous les cas, le rôle de la chirurgie nasale s'inscrit dans le cadre d'un programme thérapeutique multisite. Toutefois, la normalisation des résistances nasales représente une étape fondamentale du traitement du ronflement, contribuant à rétablir la respiration nasale physiologique en réduisant le gradient de pression trans-pharyngé, en atténuant le collapsus du segment vélo-pharyngien et en améliorant le résultat d'autres procédures chirurgicales. En outre, on ne doit pas sous-estimer le fait que certains patients acceptent de subir un acte chirurgical sur un seul site plus facilement que sur plusieurs sites.

Les techniques chirurgicales préférentielles sont selon nous, outre la correction des difformités de la cloison et de la valve nasale, celles qui comportent l' approche la moins traumatique possible sur les cornets, souvent siège principal de l'obstruction; à cette fin, deux moyens répondent le plus à nos exigences: les hautes fréquences bipolaires (H.F.) et les radiofréquences bipolaires (R.F.). Les résultats finaux sont presque superposables mais les RF semblent mieux respecter la physiologie nasale. En faveur des H.F., on retient le coût mineur de l'instrumentation.

\section{Introduction}

Environ $25 \%$ de la population adulte ronfle habituellement; l'incidence augmente dans le sexe masculin, entre 40 et 64 ans, dans le sujets avec poids excessif et en particulier dans l'obèse, dans les fumeurs ${ }^{24,30}$.

Dans l'approche diagnostique-thérapeutique au patient qui se plaint de perturbation du sommeil du degré divers, on ne peut pas négliger d'une classification et séparation claire du roncopathe pur des patients qui respondent plus proprement à une d' apnées du sommeil (OSAS). Ceci parce qu' il existe un continuum de situations entre le sujet normal et le patient atteint d' apnées nocturnes, passant par le ronfleur simple et la syndrome respiratoire des voies aériennes supérieures (UARS). Dans des conditionnes de sommeil normale, à chaque action d'inspiration on a qu'une réduction modeste des diamètres du segment coallassable sans aucun phénomène bio-mécanic signicatif ${ }^{1}$. Dans ce cas-ci il y a une réactions mio-élastiques valide vers l'extérieur opposé à la pression négative intra-luminale modeste grace à la basse résistance nasale, avec enfin une stabilité substantielle du diamètre des VAS. Dans le ronfleur chronique cet équilibre change pour trois facteur pathologiques differents: augmentation des résistances nasales avec l'abaissement conséquent de la pression négative intra-pharygienne; augmentation de l'épaisseur des tissous mou pariétales; réduction de l'activité expansible mio-élastique. 
La thérapie chirurgicale peut se diriger vers sites divers, singulièrement ou en association: le site nasal, le site oropharyngien, l'hypopharynx et le larynx mais comme on peut facilement intuire le «primum movens» est souvent l'obstruction nasale. La normalisation des résistances nasales représente une étape fondamentale du traitement du ronflement, contribuant à rétablir la respiration nasale physiologique en réduisant le gradient de pression trans-pharyngé, en atténuant le collapsus du segment vélo-pharyngien ${ }^{29}$. En outre, on ne doit pas sous-estimer le fait que certains patients acceptent de subir un acte chirurgical sur un seul site plus facilement que sur plusieurs sites ${ }^{8}$.

$\mathrm{Au}$ niveau nasal et rhino-pharyngien les problématique dismorpho-fonctionnelles, quand le traitement pharmacologique n'as pas eu résultat, peuvent être résolu par une intervention chirurgicale, en allant agir sur les diverses structures anatomiques par correction de la déviation de la cloison, valvuloplastique, réduction où ablation des cornets, ablation des végétations $^{15-19}$

Dans nôtre étude nous avons réuni l'expérience de deux Services Hôpitaliers Universitaires dans le traitement du ronflement simple d'origine nasale, pour estimer l'efficacité de la thérapie proposée afin de résoudre l'obstruction nasal et donc la roncopathie. En particulier la portée du travail a été également celle-là de comparer l'utilisation, en association avec les techniques classiques de septoplastie, de deux instruments pour la plastique des cornets, les haute fréquence (H.F.) et les radiofréquences (R.F.), soulignant les avantages, les spécificités et différences ${ }^{10}$.

\section{Matériaux et méthodes}

Tous les patients insérés dans cette étude non randomisé étaient ronfleurs chronique depuis plus de 6 mois et la somnolence était évaluée par le score à l'échelle d'Epworth (JOHNS M.W., Sleepiness in different situations measured by the Epworth Sleepness, Scale. Sleep 1994. 17(8): 703-10). Les patients qui ont présenté un score supérieur à 10 ont exécuté un enregistrement polysomnographique et, dans le cas où l'index d'apnée/hypopnée était supérieur à 10 , ont été exclus de cette étude.

Donc, tous les patients qui présentaient des apnées du sommeil ont été préventivement exclus de cet travail. Personne des patients inclus présentait d'ailleurs des anomalies anatomiques importantes des voies aériennes supérieures, comme le micrognatisme, le rétrognatisme, ni sténose hypopharyngiennes ni maladies allergiques $^{3}$. Ont été également exclus tous les sujets qui ont présenté des polypes, une sinusite chronique ou s'ils avaient déjà supporté une chirurgie nasales.
Enfin 120 patients traité dans l'arc de 6 mois (du décembre 2001 à mai 2002) ont été insérés dans l'étude: il y avais 84 mâles et 36 femelles; l'âge se situait entre 38 et 63 ans (total moyen 50.5 ans). Avant intervention tous les patients avais été conseillé à suivre un régime jusqu'à obtenir un index de la masse corporelle (BMI) $(\mathrm{Kg} / \mathrm{m})^{4}$ entre 21.4 et 30.5 .

Sur 62 patients nous avons pratiqué une septoplastie plus plastie des cornet avec H.F. et sur 58 patients la septoplastie a été associé à plastie des cornets par R.F.; toutes les intervention étaient sous anesthésie générale.

Le diagnostic pre-chirurgical de roncopathie lié à obstruction nasal a été faite en consultation par les moyen suivant: rhinoscopie classique, rhinoendoscopie avec fibres optiques $0^{\circ}$ e $45^{\circ}$, manoeuvre de Muller, rhinomanométrie, étude du temp de transport muco-ciliair (TTMC). Le ronflement ainsi que l'obstruction nasale ont été évalués de manière subjective au moyen d'une échelle visuelle analogique. Cette stadification pour le ronflement a été réalisée aussi par des questionnaires subjectif réalisés par le patient lui même et par le ou la conjointe du patient. Il y avais diverses variable:

- évaluation de la respiration nasale, avec une échelle subjective au patient $(0=$ respiration normale, 1 = difficulté respiratoire minime, $2=$ difficultés modérées, 3 = difficultés graves, $4=$ difficultés maximum).

- évaluation du ronflement (conjoint) avec une échelle en 5 niveaux (1: non ronfle; 2 : ronfle peut et le conjointe est éveillé seulement de temps en temps; 3: ronfle modérément et le conjoint est éveillé fréquemment; 4: ronfle sérieusement et le conjoint dort dans une autre salle; 5: ronfle de manière insopportable même à distance.

L'effet de la thérapie chirurgicale effectuée a été considéré positif si le score du patient amélioraient de 3-4-5 à 1-2.

Les examens et les questionnaires ont été répétés après un mois et à une année de distance de l'intervention chirurgicale. Les patients ont été divisé dans deux groupes sélon le type de technique chirurgicale utilisé: groupe A (GA) et groupe B (GB)

Les patients du GA ont été soumis au traitement chirurgical de septoplastie plus plastie des cornet avec haute fréquence. Tous les intervention ont été effectuées par chirurgien experts (skills). Sur la technique chirurgicale utilisée pour la correction de la cloison nous ne nous arrêtons pas en sachant que les aspects des techniques de Cottle et de Killian se combinent souvent tandis que plus de nouveauté il y a dans les divers instrument et utilisations d'eux pour la plastie des cornets dans nos deux services: 
-du point de vue technique la chirurgie à haute fréquence $3,9,15,19,20,23,25,26$ utilise une émission bipolaire programmée à haute fréquence qui distribue une puissance parfaitement stable sans modification dans le temps, avec controle précis de la durée de l'émission (en cent de sec.) et modulation appropriè: 1'H.F. est en fait programmable avec la possibilité d'employer second les cas plus de puissances (efficacité sûre) pendant des périodes d'action très réduit (pas lésions à tissus). La technique que nous avons employé (Mora-Barbieri) permet, par la cautéristion du cornet par "glissement" le bloc des récepteurs du sensoriel du V (afférent) et l'inibition (arc reflété) du systéme parasymphatique (efférent). L'équipement que nous avons utilisé est le HF Timed - KORPO 100 avec sonde bipolaire, avec côntrol de puissance (1$47 \mathrm{~W})$ temporisé ou manuel.

- La sonde des RF (CELONLAB ENT) par contre est introduite déjà active, afin d'éviter des microhemorrhagies, dans la tête du cornet et inserée sur toute la longueur du cornet. Les R.F. causent la vaporisation pour excitation ionique des molécules d'eau avec coagulation conséquente de la sousmuqueuse sans nécrose des muqueuses. La période d'application est commandée également avec un signal sonor, la puissance va de 1 à $25 \mathrm{~W} ; 5$ est la puissance que nous utilison habituellement. L'effet immédiat est la dénaturation des protéines, suivi par la coagulation (aspect blanchâtre) et enfin par une réaction oedemateuse (après 24 heures) dans la zone de l'application de l'électrode. Après 72 heures il y a une réaction cicatritielle qui se prolonge pendant 2 ou 3 semaines. La vaporisation est proportionelle à la puissance et l'effet sur les tissus à la période de l'application.

Tous les patients ont maintenu un méchage nasal bilatéral (Merocel) pendant 48 heures. Une thèrapie antibiotique pendant 6 jours a été commandé plus $1 \mathrm{~g}$ de codeine+paracetamolo 2 fois par jour à la demande afin d'alléger la douleur.

Le chi-test a été employé pour comparer la répartition de fréquence en âge, sexe et complications. Les tests d'analyse de variance comparée (ANOVA) et le t-test ont été utilisés pour comparer les deux groups. Les différences ont été considérées statistiquement signicatives si $\mathrm{p}<0.05$.

\section{Résultats}

Nous avons analysé et comparé les paramètres suivants:

- Temp de transport muco-ciliair (TTMC)

- rhinomanomètrie (résistances nasaux totaux)

- durée de l'intervention chirurgicale
- échelles d'évaluation subjective de l'obstrction nasal et du ronflement

- complications

Patients du Group A: ceux-ci ont eu tous une réduction des résistances nasaux enregistrés par la rhynomanomètrie (Graphique 1)

Les paramètres du TTMC sont résumées dans le Tableau II.

La durée de moyenne de l'intervention chirurgicale a été de $21 \mathrm{~min}$. environ.

Patients du Group B: ceux-ci également ont présenté une réduction des résistances à la rhinomanomètrie soit après un mois soit à un année de distance (Graphique 1).

Les paramètres du TTMC sont résumées dans le Tableau II.

La durée de moyenne de l'intervention chirurgicale a été de $24 \mathrm{~min}$. environ.

Le score de l'échelle d'évaluation subjective de la respiration nasale et du ronflement a baissé de manière signicative dans le deux groupes sans différences signicatives entre les deux techniques (Graphique 2 et 3 ) mais de manière signicative si confronté aux paramètres enregistrés avant le traitement chirurgicale; cette amélioration ont restés bonnes aussi à une année de distance. Les différences ont été considérées comme statistiquement significatives lorsque $\mathrm{p}<0.05$. Les complications à court terme dans les premiers 30 jours ont été rapportés dans le (Graphique 3).

Tous les patients ont maintenu un index de masse corporel (BMI) superposable à celui de avant l'intervention.

Il n'existait aucune différence significative entre les deux groupes en ce qui concerne le grade initial du ronflement, l'index d'Epworth, le sexe, l'age, l'index de masse corporel (BMI) ainsi que les résultats des échelles d'evaluation subjective de l'obstruction nasale (SVO) et du ronflement (SVR) à un année de l'intervention (Tableau I).

Tableau I: analyse de population $(S D=$ standard deviation; NS = non significatif $(p>0.05)$; SVR = score valutatif du ronflement SVO = score valutatif de l'obstruction nasal)

\begin{tabular}{|c|c|c|c|}
\hline & GA & GB & GA vs GB \\
\hline \multicolumn{3}{|l|}{ age } & NS \\
\hline moyenne & 46,7 & $54, .2$ & \\
\hline SD & 10,6 & 8,7 & \\
\hline \multicolumn{3}{|l|}{ Sexe } & NS \\
\hline hommes & 43 & 41 & \\
\hline femmes & 20 & 16 & \\
\hline \multicolumn{3}{|l|}{ BMI (a J0) } & NS \\
\hline
\end{tabular}




\begin{tabular}{|l|r|r|l|}
\hline index & 24,7 & 26,4 & \\
\hline DS & 5,5 & 5,1 & \\
\hline SVR (à J 360) & 1,9 & 1,8 & NS \\
\hline moyenne & 1,46 & 1,1 & \\
\hline SD & & NS \\
\hline Epworth (à J0) & 4,76 & 4,68 & \\
\hline moyenne & 3,24 & 2,65 & \\
\hline SD & 1 & 1.2 & NS \\
\hline SVO (à J 360) & 0,66 & 0,15 & \\
\hline moyenne & & & \\
\hline SD &
\end{tabular}

Tableau II: TTMC: les données sont exprimées en minutesi (moyenne $\pm \mathrm{DS}$ ). Valeur normal de $13 \pm 2$ minutes. $\mathrm{N}=$ numero de patients (numero réel exclus ce qui ont été perdu au follow-up).

\begin{tabular}{|l|c|c|}
\hline \multicolumn{1}{|c|}{ Follow-up } & $\mathrm{A}=\mathrm{N}=\mathbf{6 2}$ & $\mathrm{B}=\mathrm{N}=\mathbf{5 8}$ \\
\hline J0 Avant chirurgie & $15 \pm 2.45(62)$ & $15 \pm 2.15(58)$ \\
\hline $\mathrm{J} 7$ & $14 \pm 1.67(59)$ & $14 \pm 2.13(56)$ \\
\hline $\mathrm{J} 30$ & $12 \pm 2.15(50)$ & $13 \pm 1.14(38)$ \\
\hline $\mathrm{J} 360$ & $12 \pm 2.41(31)$ & $12 \pm 3.1(22)$ \\
\hline
\end{tabular}

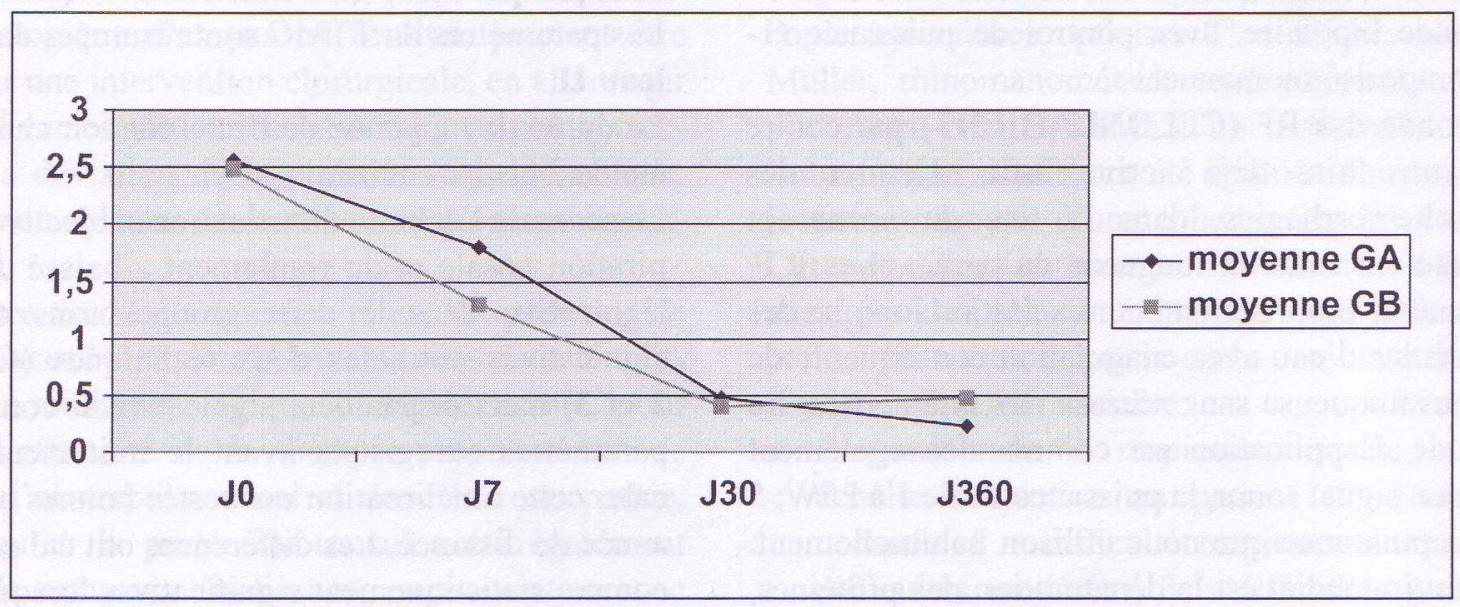

Graphique 1: Rhinomanomètrie antérieure: moyenne des resistances nasaux totaux avant et après chirurgie (J 7-J 30- J 360) en Pasc/cm3 (GA = 62 patientsi; GB = 58 patients).

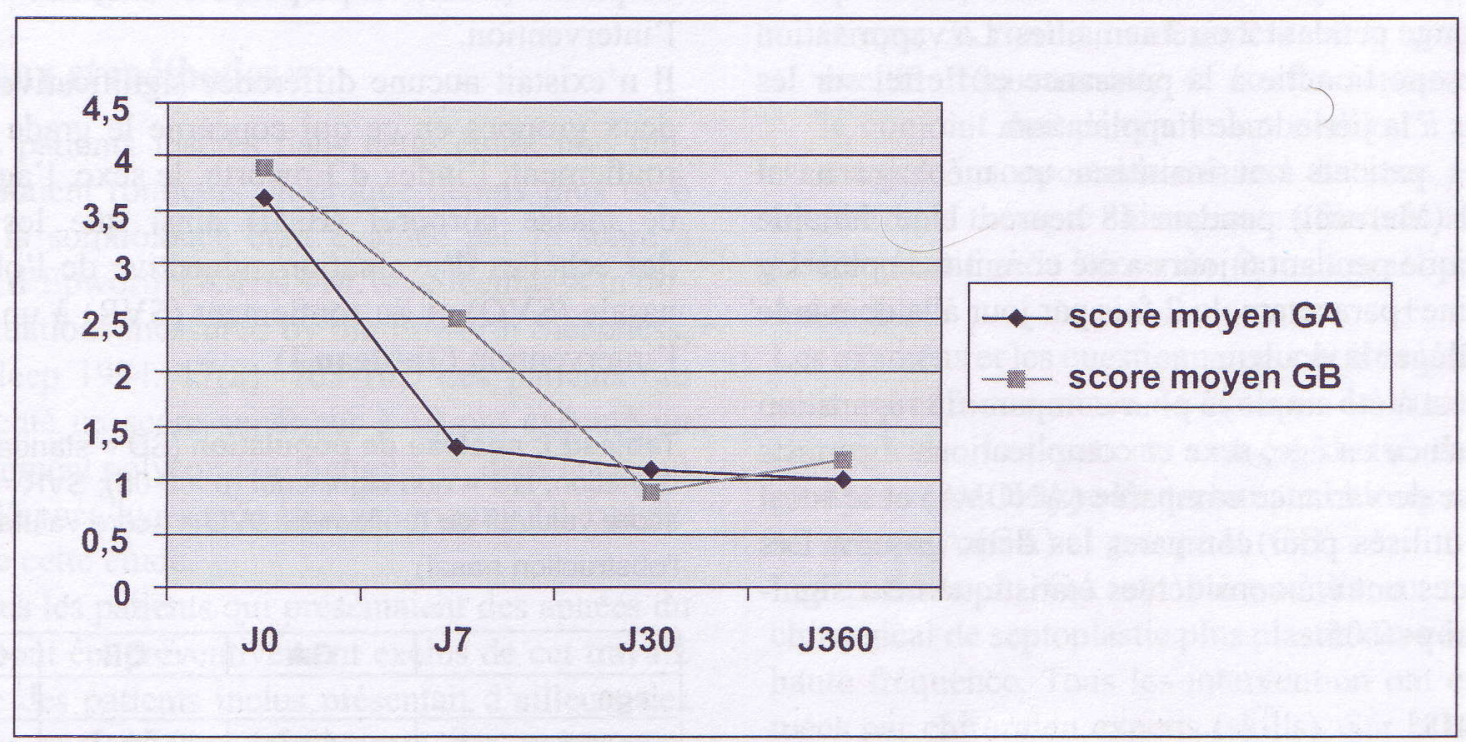

Graphique 2: Questionnaire d'évaluation subjectif de l'obstruction nasal: avant et après chirurgie ( $\mathrm{J} 7, \mathrm{~J} 30$ et J 360). Donnés moyenns pour le GA (62 patients) et pour le GB (58 patients) $(0=$ respiration normale, 1 = difficulté respiratoire minime, 2 = difficultés modérées, 3 = difficultés graves, 4 = difficultés maximum). 


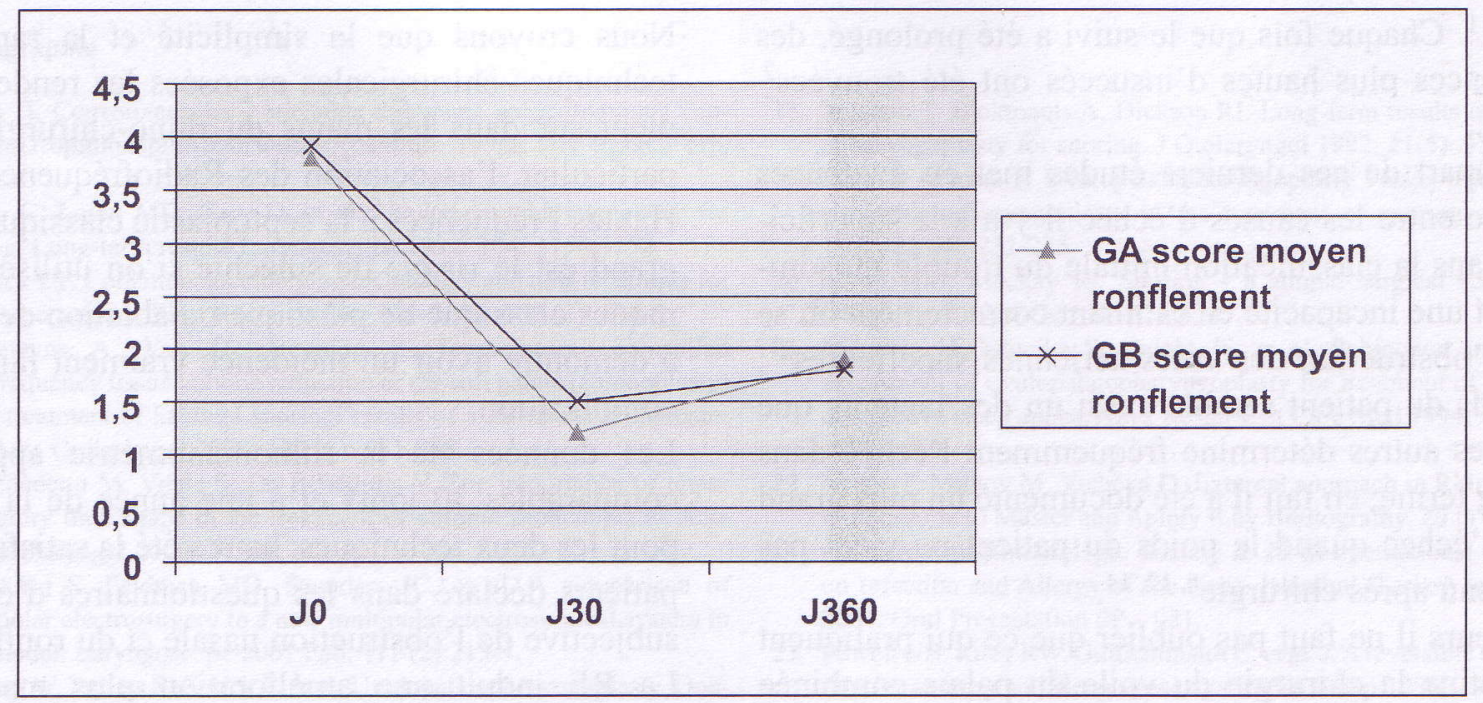

Graphique 3: Questionnaire d'évaluation subjectif du ronflement: donnés moyen avant et après (J0, J30, J 360) (1: non ronfle; 2 : ronfle peut et le conjointe est éveillé seulement de temps en temps; 3: ronfle modérément et le conjoint est éveillé fréquemment; 4: ronfle sérieusement et le conjoint dort dans une autre salle; 5 : ronfle de manière insopportable même à distance).

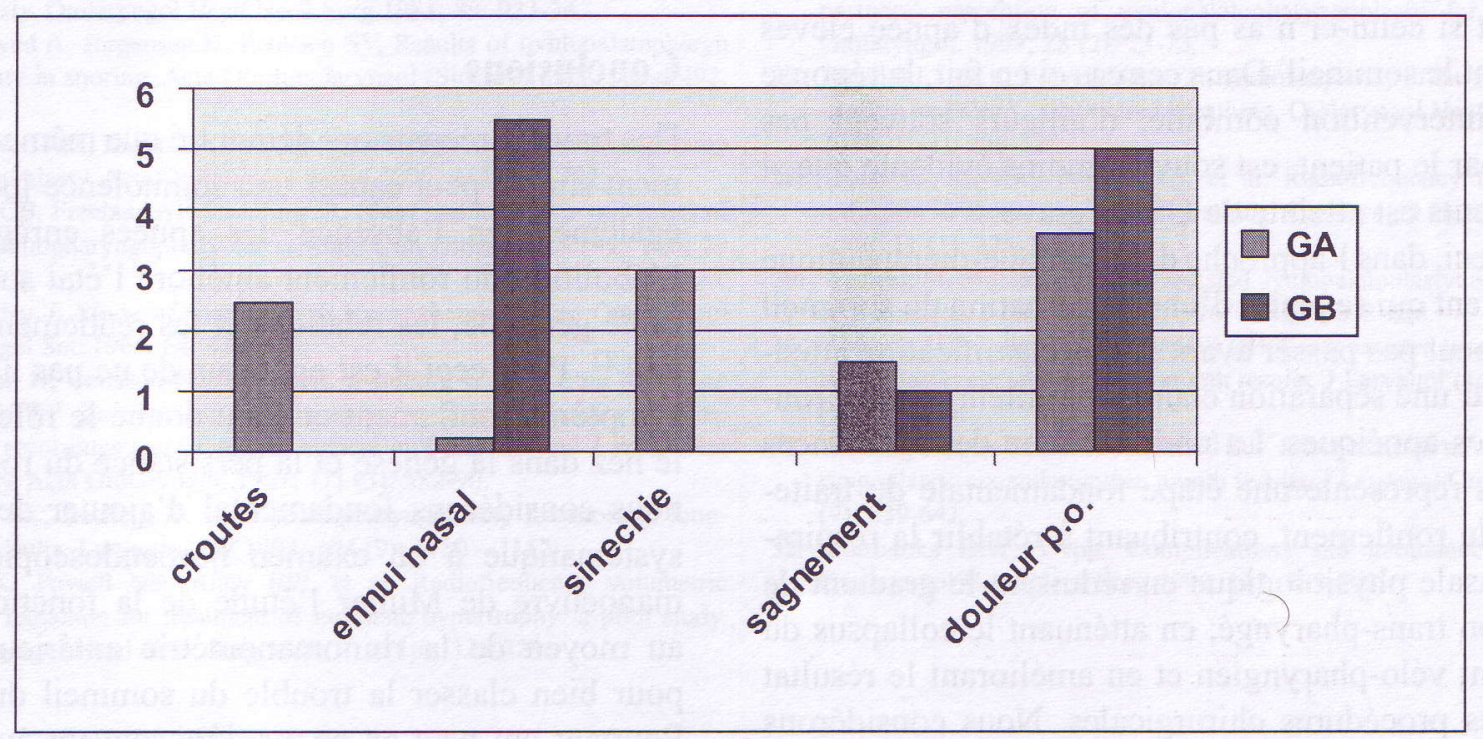

Graphique 4: Complications et douleur post-chirurgicale (p.c.): \% sur le numero total de patients traités ( $\mathrm{GA}=62$ patients, $\mathrm{GB}=58$ patients).

\section{Discussione}

Dans ces dernières années beaucoup a été discuté en littérature la vraie efficacité des thérapies chirurgicales proposées pour traiter la roncopathie; en fait des données de la littérature on sait que seulement 55$71 \%$ des patients qui ont été traité chirurgicalement sur un seul site ont démontre une amélioration des symptômes pendant le sommeil dans le long periode ${ }^{4}$, $12,13,15,17,24,29$
Miljeteig conclut tout à fait que dans le long terme il n'y a aucune différence signicative entre les données objectifs de la roncopathie avant et après chirurgie même si les patients se déclarent satisfait dans 79\% des cas, faisant supposer un effet placebo important de la chirurgie. ${ }^{19}$

Il est d'autre part vrai que la plupart des données de la littérature sur des techniques chirurgicales susceptibles de corriger le roncopathie ont un suivi à court 
terme ${ }^{11}$. Chaque fois que le suivi a été prolongé, des fréquences plus hautes d'insuccés ont été trouvées ${ }^{2}$, $6,14,17$

La plupart de ces derniers études met en évidences comme entre les causes d'échec il y a une superficialité dans la classification initiale du trouble du sommeil et une incapacité en estimant correctement ou se situé l'obstruction des voies aériennes supérieures ${ }^{21}$; le poids du patient semble aussi un des facteurs que plus des autres détermine fréquemment l'échec dans le long terme; en fait il a été documenté un plus grand taux d'échec quand le poids du patient ne vient pas maintenu après chirurgie $\mathrm{e}^{6,12,14}$.

D'ailleurs il ne faut pas oublier que ce qui pratiquent de routine la chirurgie du voile du palais combinée avec la chirurgie nasale, ont décrit des problèmes importants de «compliance» du patient en raison de l'oedème pharyngien concomitant au méchage nasale ${ }^{17,2}$.

Pour toutes ces raisons et notre expérience il vaut mieux s'approcher avec prudence au patient ronfleur, surtout si celui-ci n'as pas des index d'apnée élevés pendant le sommeil. Dans ces cas ci en fait, la réponse à un intervention combiné, d'ailleurs souvent pas aimé par le patient, est souvent moins évidente que si le patients est atteinte de OSAS grave.

Pour ceci, dans l'approche diagnostique-thérapeutique du patient qui se plaint d'une perturbation du sommeil on ne peut pas passer avant d' une classification attentive et d'une séparation claire du ronfleur pur des roncopathes-apnéiques. La normalisation des résistances nasales représente une étape fondamentale du traitement du ronflement, contribuant à rétablir la respiration nasale physiologique en réduisant le gradient de pression trans-pharyngé, en atténuant le collapsus du segment vélo-pharyngien et en améliorant le résultat d'autres procédures chirurgicales. Nous considérons donc fondamental pour une correcte sélection prechirurgicale d'ajouter de manière systématique à un examen fibroendoscopique avec manoeuvre de Muller l'étude de la fonction nasale au moyen de la rhinomanométrie antérieure active.

Des patients bien sélectionés comme ceux qui ont été insèré dans cette étude, s'avantagent d'une approche exclusive de l'obstruction nasal avec meilleure «compliance» et mineure incidence de complications ${ }^{1}$.

Du bout ne va pas oublié la possibilité de approcher également le voile ou d'autre sites d'obstruction avec des techniques mininvasives en anesthésie locale, quand il est indiqué et quand le site nasal n'est pas le seule identifié, par une étude appropriée, comme responsable du ronflement.
Nous croyons que la simplicité et la rapidité des techniques chirurgicales exposées les rendent instrument sur dans les mains du rhino-chirurgien et, en particulier, l'association des Radiofréquences ou des Hautes Fréquences à la septoplastie classique, où plus grand est le risque de sinéchie si on utilise les techniques classique de plastique ou ablation des cornets, a démontré avoir un incidence vraiment faible de tel complication.

Les données de la rhinomanomètrie apparaissent comparables 30 jours et à une année de la chirurgie pour les deux techniques, haut a été la satisfaction des patients déclaré dans les questionnaires d'évaluation subjective de l'obstruction nasale et du ronflement.

La RF induit une amélioration plus marquée du transport mucociliaire mais tous les deux techniques semblent conservatrices sur la normale physiologie de la muqueuse nasale.

Sur l'évaluation des frais d'exploitation il est préférable l'H.F., parce que il emploie une sonde stérilisable, concernant le RF qui utilise des sondes jetables.

\section{Conclusions}

Des travaux récents ont démontré que même le ronflement simple peut causer une somnolence journaliere, également en l'absence des apnées enrégistrables. L'abolition du ronflement améliore l'état social et de santé générale, les relations et les rendements au travail $^{22}$. Pour ceci il est opportun de ne pas négliger le symptôme ronflement et etant donné le rôle joué par le nez dans la genèse et la persistance du ronflement, nous considérons fondamental d'ajouter de manière systématique à un examen fibroendoscopique avec manoeuvre de Muller l'étude de la fonction nasale au moyen de la rhinomanomètrie antérieure active pour bien classer la trouble du sommeil du patient. Pourtant ont peut en ce maniére souvent éviter étant excessivement agressifs sur le patient lui-même avec des intervention combinées trop compliquées et douloureux. La desobstruction nasale par déplacement des courbes anormales de la cloison, des eperons ou des hypertrophies muqueuses des cornets reussit, dans les justes indications, à redonner un correct flux aérien nocturne suffisant à rééquilibrer le difficile status du sommeil.

Nos résultats dans les deux groupes traitées sont semblables en ce qui concerne la réduction des résistances nasales et l'amélioration subjective de la respiration et du ronflement. Négligeables nous paraissent les complications et la douleur enregistrée. 


\section{Bibliographie}

1. Недев П. Септопластика - модерна хирургия на девиирания септум.сп.Оториноларингологични страници. 1996г. Год ІІІ,бр.2 стр. 10-12.

2. Barry C, Levin MD, Gary D, et al. Uvulopalatopharyngoplasty for snoring. Long-term results Laryngoscope 1994; 104: 1150-1152.

3. Bortnick DP. Coblation: an emerging technology and new technique for soft-tissue surgery. Plast Reconstr Surg Feb 2001; 107 (2): 614-5

4. Boudewyns A, Van De Heyning P. Temperature- controlled radiofrequency tissue volume reduction of the soft palate (somnoplasty) in the treatment of habitual snoring: results of a European multicenter trial. Acta Otolaryngol 2000; 120 (8): 981-985.

5. De Benedetto M, Vitale S, De Benedittis V. The importance of upper respiratory tract shape in the treatment of chronic rhonchopathy. Acta Otorhinolaryngol 2000; 20 (4): 245-9.

6. Chinpairoj S, Feldman MD, Saunders JC, et al. A comparison of monopolar electrosurgery to a new multipolar electrosurgical system in a rat model. Laryngoscope $2001 \mathrm{Feb} ; 111$ (2):213-7.

7. Conway W, Fujitas S, Zorick F, et al. Uvulopalatopharyngoplasty. Oneyear followup. Chest. 1985 Sep; 88 (3): 385-7.

8. Croft CB, Golding-Wood DG. Users and complications of uvulopalato pharyngoplasty. J Laryngol Otol. 1990; 104: 871-875.

9. Ellis PDM, Williams J, Shneerson JM. Surgical relief of snoring due to palatal flutter: a preliminary report. Ann R.C.S. Engl. 1993, 75: 286290.

10. Fujiita S, Conway WA, Zorik F. Surgical correction of anatomic abnormalities in obstructive sleep apnea syndrome. Uvulopharyngopal atoplasty. Otolaryngol Head Neck Surg 1981; 89: 923-34.

11. Grontved A, Jorgensen K, Petersen SV. Results of uvulopalatopharyn goplasty in snoring. Acta Otorhinolaryngol (Stockhl) 1992; suppl 492: 11-14.

12. Grontved AM, Karup P. Complaints and satisfaction after uvulopalatop haryngoplasty. Acta Otolaryngol Suppl 2000; 543: 190-192.

13. Koay CB, Freeland AP, Stradling JR. Short and long term outcomes of uvulopaltopharyngoplasty for snoring. Clin Otolaryngol 1995 Feb; 20 (1): $45-8$.

14. Ikematsu T. Study of snoring. 4 th report therapy. J Jpn Otol Rhinol Laryngol Soc 1964; 64: 434-435.

15. Larsson H, Carlsson-Nordlander B, Svanborg E Long-time follow-up after UPPP for obstructive sleep apnea syndrome. Results of sleep apnea recordings and subjective evaluation 6 months and 2 years after surgery. Acta Otolaryngol. 1991; 111 (3): 582-90.

16. Levin BC, Beckerg D. Uvulopalatopharyngoplasty for snoring: longterm results. Laryngoscope 1994; 104 (9): 1150 - 1152.

17. Li KK, Powell NB, Riley RW, et al. Radiofrequency volumetric tissue reduction for treatment of turbinate hypertrophy: a pilot study. Otolaryngol Head Neck Surg 1998; 119 (16): 325-329.
18. Macnab T, Blokmanis A, Dickson RI. Long-term results of uvulopalat opharyngoplasty for snoring. J Otolaryngol 1992; 21(5): 350-354.

19. Maw J, Marsan J. Uvulopalatopharyngoplasty versus laser - assisted uvulopalatopharyngoplasty in the treatment of snoring. Otolaryngol 1997, 26 (4): 232-235.

20. Myatt HM, Mackay IS. Snoring - a simple surgical solution. Clin Otolaryngol 1996 Oct; 21: 419-424.

21. Milijeteg H, Mateika S, Haight JS, et al. Subjective and objective assessment of uvulopalatopharyngoplasty for treatment of snoring and obstructive sleep apnea. Am J Respir Crit Care Med 1994; 150 (5 pt 1): 1286-1289.

22. Nedev P, Milkov M, Vicheva D, External approach in Rhinoplasry - It is a possible to Master and Aplply it by Bibliografhy. 20 th Congress of the European Rhinolologic Society \& 23 th International Symposium on Infection and Allergy of the Nose. Istambul, Turkey June $18-25$ 2004. Oral Presentation 0P - 081.

23. Powell NB, Riley RW, Guilleminault C, et al. J. A reversible uvulopalatal flap for snoring and sleep apnea syndrome. Sleep 1996; 19: 593-599.

24. Simmons FB, Guilleminault C, Miles LE. The palatopharyngoplasty operation for snoring and sleep apnea: an interim report. Otolaryngol Head Neck Surg. 1984; 92 (4): 375-380.

25. Taliaferro C. Submucosal radiosurgical uvulopalatoplasty for the treatment of snoring: is the monitoring of tissue impedance and temperature necessary? Otolaryngol Head Neck Surg 2001; 124 (1): 46-50.

26. Tytherleigh MG, Thomas MA, Connolly AA, et al. Patient's and partners' perception of uvulopalatopharyngoplasty for snoring. J Otolaryngol. 1999; 28 (2): 73-75

27. Troell RJ, Li KK, Powell NB, et al. Radiofrequency of the soft palate in snoring and sleep - disordered breathing. Otolaryngol Head Neck Surg 2000; 11(1): 226-228.

28. Troell RJ, Li KK, Powell NB, et al. Radiofrequency tongue base reduction in sleep- disordered breathing. Otolaryngol Head Neck Surg 2000; 11 (1): 375-377.

29. Wareing M, Mitchell D. Laser assisted uvulopalatoplasty: an assesment of a tecnique. J Laryngol Otol 1996; 110 (3): 232-236.

30. Wareing MJ, Callanan VP, Mitchell DB. Laser assisted uvulopalatopha ryngoplasty: six and eighteen month results. J Laryngol otol 1998; 112 (7): 639-641.

31. Wareing MJ, Callanan VP, Mitchell DB. Laser assisted uvulopalatopha ryngoplasty: six and eighteen month results. J Laryngol Otol 1998; 112 (7): 639-641.

32. Wairbanks DNF. Uvpp. Complications and avoidance strategies. Otolaryngol Head Neck Surg 1990; 102: 239-245. 